\title{
Unital Four-Dimensional Lattice-Ordered Algebras with a Distributive Basis
}

\author{
Mustafa Akkurt, ${ }^{1}$ Zainab Alshair ${ }^{2}$ \\ Lena Lamar, ${ }^{2}$ and Jingjing $\mathbf{M a}^{2}$ \\ ${ }^{1}$ Department of Mathematics, Gebze Institute of Technology, 41400 Kocaeli, Turkey \\ ${ }^{2}$ Department of Mathematics, University of Houston-Clear Lake, 2700 Bay Area Boulevard, Houston, \\ TX 77058, USA
}

Correspondence should be addressed to Jingjing Ma, ma@uhcl.edu

Received 5 May 2011; Accepted 2 June 2011

Academic Editors: V. De Filippis, W. Gao, and T. Nakatsu

Copyright (C) 2011 Mustafa Akkurt et al. This is an open access article distributed under the Creative Commons Attribution License, which permits unrestricted use, distribution, and reproduction in any medium, provided the original work is properly cited.

All unital four-dimensional lattice-ordered algebras with a $d$-basis are constructed.

\section{Introduction}

Lattice-ordered algebras ( $\ell$-algebras) with a distributive basis ( $d$-basis) were studied in [5] in an effort to obtain general structure theory for a class of $\ell$-algebras that includes some well-known and important examples of $\ell$-algebras, such as matrix and triangular matrix $\ell$ algebras over a totally ordered field with entrywise lattice order, polynomial $\ell$-algebra over a totally ordered field with coordinatewise lattice order, and so forth.

All unital two-dimensional and three-dimensional $\ell$-algebras with a $d$-basis have been constructed [6]. In this paper, we construct all unital four-dimensional $\ell$-algebras with a $d$ basis. We will first review some definitions and results for later use. The reader is referred to $[1,5,6]$ for the general theory of lattice-ordered rings ( $\ell$-rings) and undefined terminologies in this paper. Throughout $F$ always denotes a totally ordered field.

Let $R$ be an $\ell$-ring. The positive cone of $R$ is $R^{+}=\{r \in R: r \geq 0\}$. A vector lattice $V$ over $F$ is an $\ell$-group and a vector space over $F$ such that $F^{+} V^{+} \subseteq V^{+}$. An $\ell$-algebra $A$ over $F$ is an algebra over $F$, that is, an $\ell$-ring and a vector lattice over $F$. An $\ell$-algebra is called unital if it has an identity element 1 and called $\ell$-unital if $1 \geq 0$. An element $a$ in an $\ell$-ring is called basic if $a>0$ and, for any $b, c \geq 0, a \geq b, c$ implies that $b$ and $c$ are comparable, that is, either $b \geq c$ or $c \geq b$. A set of elements $\left\{x_{i}: i \in I\right\}$ is called disjoint if each $x_{i}>0$ and $x_{i} \wedge x_{j}=0$ for 
any two different elements $x_{i}$ and $x_{j}$. An element $x \geq 0$ in an $\ell$-ring $R$ is called an $f$-element if $y \wedge z=0$ implies that $x y \wedge z=y x \wedge z=0$, and $x$ is called a d-element if $y \wedge z=0$ implies that $x y \wedge x z=y x \wedge z x=0$. An $\ell$-ring $R$ is called $\ell$-reduced if for any $a \in R^{+}, a^{2}=0$ implies that $a=0$.

Let $A$ be an $\ell$-algebra over $F$. A nonempty subset $S$ of $A$ is called a $d$-basis of $A$ if $S$ is a disjoint subset of $A$ that spans $A$ as a vector space over $F$ and each element in $S$ is a $d$-element [6, Definition 2.1]. Then for each $a \in A, a=\alpha_{1} s_{1}+\cdots+\alpha_{p} s_{p}$, where $p \geq 1, \alpha_{1}, \ldots, \alpha_{p} \in F$, and $s_{1}, \ldots, s_{p} \in S$ are distinct, and $a \geq 0$ if and only if each $\alpha_{i} \geq 0, i=1, \ldots, p$. Thus in a $d$-basis, each element is basic. Let $A$ be a unital finite-dimensional $\ell$-algebra with a $d$-basis. Then $A$ is $\ell$-unital [1], and there exists a $d$-basis $S$ such that $1=e_{1}+\cdots+e_{n}$, where $e_{1}, \ldots, e_{n} \in S$ are distinct.

In the following theorem, we record some results on unital $\ell$-algebras with a $d$-basis from $[5,6]$.

Theorem 1.1. Let $A$ be a unital finite-dimensional $\ell$-algebra over $F$ with a d-basis $S$ such that $1=$ $e_{1}+\cdots+e_{n}$, where $n \geq 1$ and $e_{1}, \ldots, e_{n} \in S$ are distinct.

(1) Each $e_{i}$ is an idempotent $f$-element and $e_{i} e_{j}=0$ for $i \neq j$.

(2) Let $a$ be a basic element. Then there exists exactly one $e_{i}$ such that $e_{i} a=a$, and for any $j \neq i, e_{j} a=0$. Similarly, there exists exactly one $e_{k}$ such that $a e_{k}=a$, and for any $j \neq k$, $a e_{j}=0$.

(3) For each element $a \in S$, if $e_{i} a=a$ or $a e_{i}=a, 1 \leq i \leq n$, then either $a$ is nilpotent or $a^{n_{a}}=\alpha_{a} e_{i}$ for some positive integer $n_{a}$ and $0<\alpha_{a} \in F$.

(4) Let $a, b \in S$. If either $a$ or $b$ is not nilpotent and $a b \neq 0$, then $a b$ is basic.

\section{The Classification of Unital Four-Dimensional $\ell$-Algebras with a $d$-Basis}

We first list some $\ell$-algebras that we are going to use for the classification of unital fourdimensional $\ell$-algebras over $F$ with a $d$-basis.

(i) Let $G$ be a finite group, and let $F[G]$ be the group algebra over $F$. Define an element $\sum_{i} \alpha_{i} g_{i} \geq 0$ if each $\alpha_{i} \geq 0$. Then $F[G]$ is an $\ell$-algebra over $F$ and $G$ is a $d$-basis. In the following, $F[G]$ always denotes the $\ell$-algebra with the lattice order defined above.

(ii) Let $0<\alpha \in F$, and let $f(x)=x^{n}-\alpha \in F[x]$ be irreducible. We use $a=\sqrt[n]{\alpha}$ to denote a fixed root of $f(x)$. Consider the extension field $F[\sqrt[n]{\alpha}]$ of $F$. Then

$$
F[\sqrt[n]{\alpha}]=\left\{\alpha_{n-1} a^{n-1}+\cdots+\alpha_{1} a+\alpha_{0} \mid \alpha_{i} \in F\right\}
$$

and $\left\{a^{n-1}, \ldots, a, 1\right\}$ is a basis of $F[\sqrt[n]{\alpha}]$ over $F$ as a vector space over $F$. Now define $\alpha_{n-1} a^{n-1}+\cdots+\alpha_{1} a+\alpha_{0} \geq 0$ if each $\alpha_{i} \geq 0, i=0,1, \ldots, n-1$. Since $a^{n}=\alpha>0$, $F[\sqrt[n]{\alpha}]$ becomes an $\ell$-algebra over $F$ with respect to the above lattice order, and $a$ is a $d$-element. Thus $\left\{a^{n-1}, \ldots, a, 1\right\}$ is a $d$-basis of the $\ell$-algebra $F[\sqrt[n]{\alpha}]$ over $F$. In this paper, $n \leq 4$ and $F[\sqrt[n]{\alpha}]$ always denotes the $\ell$-algebra defined above.

(iii) For $n \geq 1$, let $M_{n}(F)$ and $T_{n}(F)$ be $n \times n$ matrix algebra and upper triangular matrix algebra, respectively. Define a matrix positive if each of its entries is positive. Then 
$M_{n}(F)$ and $T_{n}(F)$ become $\ell$-algebras. Let $e_{i j}$ denote the matrix with $i j$ th entry equal to 1 and other entries equal to 0 . Then $\left\{e_{i j} \mid 1 \leq i, j \leq n\right\}$ is a $d$-basis for $M_{n}(F)$ over $F$ and $\left\{e_{i j} \mid 1 \leq i \leq j \leq n\right\}$ is a $d$-basis for $T_{n}(F)$ over $F$. In the following, $M_{n}(F)$ and $T_{n}(F)$ always denote the $\ell$-algebras with the above lattice order.

All nonisomorphic unital four-dimensional $\ell$-algebras with a $d$-basis are listed in Theorem 2.1. Suppose that $A$ is a unital four-dimensional $\ell$-algebra with a $d$-basis $S$ such that 1 is a sum of some distinct elements in $S$. In the proof of Theorem 2.1, we consider four different cases according to the number of elements in this sum, and in each case we consider two cases in which $A$ is $\ell$-reduced and $A$ is not $\ell$-reduced, respectively. We use $\oplus$ to denote a direct sum of $\ell$-rings.

Suppose that $A$ is a four-dimensional $\ell$-algebra with a $d$-basis $S=\left\{e_{1}, e_{2}, e_{3}, e_{4}\right\}$ such that $1=e_{1}+e_{2}+e_{3}+e_{4}$. By Theorem 1.1, each $e_{i}$ is an idempotent $f$-element, and for $1 \leq$ $i, j \leq 4, i \neq j, e_{i} e_{j}=0$. Thus $A \cong F \oplus F \oplus F \oplus F$ is a four-dimensional $f$-algebra. This is (1) in Theorem 2.1.

In the following sections, we consider the remaining three cases where 1 is a sum of three, two, or one element from the $d$-basis $S$, respectively.

Theorem 2.1. Let $A$ be a unital four-dimensional $\ell$-algebra over $F$ with a d-basis. Then $A$ is isomorphic to one of following $\ell$-algebras over $F$ :

(1) $F \oplus F \oplus F \oplus F$,

(2) $F[G] \oplus F \oplus F$, where $G$ is a group of order two,

(3) $F[\sqrt{\alpha}] \oplus F \oplus F$, where $0<\alpha \in F$,

(4) $(F 1+F a) \oplus F \oplus F$, where $a^{2}=0$,

(5) $T_{2}(F) \oplus F$,

(6) $F[G] \oplus F[G]$, where $G$ is a group of order two,

(7) $F[G] \oplus F[\sqrt{\alpha}]$, where $0<\alpha \in F$ and $G$ is a group of order two,

(8) $F[\sqrt{\alpha}] \oplus F[\sqrt{\beta}]$, where $0<\alpha, \beta \in F$,

(9) $F[G] \oplus F$, where $G$ is a group of order three,

(10) $F[\sqrt[3]{\alpha}] \oplus F$, where $0<\alpha \in F$,

(11) $M_{2}(F)$,

(12) $(F 1+F a+F b) \oplus F$, where $a^{2}=b^{2}=a b=b a=0$,

(13) $(F 1+F a) \oplus(F 1+F b)$, where $a^{2}=b^{2}=0$,

(14) $T_{2}(F)+F a$, where $e_{11} a=a e_{11}=a, a e_{12}=0$, and $a^{2}=0$,

(15) $T_{2}(F)+F a$, where $e_{11} a=a e_{22}=a$, and $a^{2}=0$,

(16) $T_{2}(F)+F a$, where $e_{22} a=a e_{11}=a, e_{12} a=a e_{12}=0$, and $a^{2}=0$,

(17) $T_{2}(F)+F a$, where $e_{22} a=a e_{22}=a, e_{12} a=0$, and $a^{2}=0$,

(18) $\left(F 1+F a+F a^{2}\right) \oplus F$, where $a^{3}=0$,

(19) $F[G] \oplus(F 1+F a)$, where $a^{2}=0$,

(20) $F[\sqrt{\alpha}] \oplus(F 1+F a)$, where $a^{2}=0$ and $0<\alpha \in F$,

(21) $F[G]$, where $G$ is a cyclic group of order four,

(22) $F[\sqrt[4]{\alpha}]$, where $0<\alpha \in F$, 
(23) $F[G]+F[G] b$, where $G=\{e, a\}$ is a group of order two and $b^{2}=\alpha a$ with $0<\alpha \in F$ and $\sqrt{\alpha} \notin F$,

(24) $F[G]$, where $G$ is the Klein four group,

(25) $F[\sqrt{\alpha}][G]$, where $0<\alpha \in F$ and $G$ is a group of order two,

(26) $F[\sqrt{\alpha}][\sqrt{\beta}]$, where $0<\alpha, \beta \in F, \sqrt{\beta} \notin F[\sqrt{\alpha}]$,

(27) $F 1+F a+F b+F c$, where $a^{2}=b+c$ and the product of any other two elements of $a, b, c$ is zero,

(28) $F 1+F a+F a^{2}+F a^{3}$, where $a^{4}=0$,

(29) $F 1+F a+F a^{2}+F b$, where $b^{2}=\alpha a^{2}, 0<\alpha \in F$, and the product of any two elements not both $a$ or $b$ is zero,

(30) $F 1+F a+F a^{2}+F b$, where the product of any two elements not both $a$ is zero,

(31) $F 1+F a+F b+F c$, where $F a+F b+F c$ is a zero ring,

(32) $F 1+F a+F b+F(a b)$, where $a^{2}=b^{2}=b a=0$,

(33) $F 1+F a+F b+F(a b)$, where $a^{2}=b^{2}=0$ and $b a=\alpha(a b)$ with $0<\alpha \in F$,

(34) $F[G]+F[G]$, where $G$ is a group of order two, $a^{2}=0$, and a commutes with each element in $F[G]$,

(35) $F[\sqrt{\alpha}]+F[\sqrt{\alpha}] a$, where $a^{2}=0$ and a commutes with each element in $F[\sqrt{\alpha}]$.

\section{1 Is a Sum of Three Disjoint Basic Elements}

Let $A$ be a unital four-dimensional $\ell$-algebra with a $d$-basis $S=\left\{e_{1}, e_{2}, e_{3}, a\right\}$ such that $1=$ $e_{1}+e_{2}+e_{3}$. Then by Theorem 1.1, each $e_{i}$ is an idempotent $f$-element, $i=1,2,3$, and $e_{i} e_{j}=0$ for any $1 \leq i, j \leq 3, i \neq j$. By Theorem 1.1, we may assume that $e_{1} a=a$ and $e_{2} a=e_{3} a=0$. We consider two cases according to whether $A$ is $\ell$-reduced or not.

\subsection{A Is $\ell$-Reduced}

If $a e_{1}=0$, then $a e_{i}=a$ for $i=2$ or 3 by Theorem 1.1(2), and hence $a^{2}=\left(a e_{i}\right)\left(e_{1} a\right)=0$, which contradicts the fact that $A$ is $\ell$-reduced. Thus $a e_{1}=a$ and $a e_{2}=a e_{3}=0$. Since $a$ is not nilpotent, by Theorem 1.1(4), $a^{2}$ is a basic element, so $a^{2}=\alpha s$ for some $0<\alpha \in F$ and $s \in S$. Since $e_{1} \wedge a=0$ and $e_{1} a=a, s \neq a$. If $s=e_{2}$ or $e_{3}$, then $a^{3}=(\alpha s) a=\alpha(s a)=0$, which is a contradiction. Thus $a^{2}=\alpha e_{1}$ for some $0<\alpha \in F$. There are two cases now.

(1) There exists $0<\beta \in F$ such that $\beta^{2}=\alpha$.

Let $b=a / \beta$. Then $\left\{e_{1}, e_{2}, e_{3}, b\right\}$ is also a $d$-basis with the following multiplication table:

\begin{tabular}{c|cccc} 
& $e_{1}$ & $b$ & $e_{2}$ & $e_{3}$ \\
\hline$e_{1}$ & $e_{1}$ & $b$ & 0 & 0 \\
$b$ & $b$ & $e_{1}$ & 0 & 0 \\
$e_{2}$ & 0 & 0 & $e_{2}$ & 0 \\
$e_{3}$ & 0 & 0 & 0 & $e_{3}$
\end{tabular}

Thus $A \cong F[G] \oplus F \oplus F$, where $G$ is a group of order two. This is (2) in Theorem 2.1. 
(2) The polynomial $f(x)=x^{2}-\alpha \in F[x]$ has no root in $F$.

Then we have the following multiplication table for $S$ :

\begin{tabular}{c|cccc} 
& $e_{1}$ & $a$ & $e_{2}$ & $e_{3}$ \\
\hline$e_{1}$ & $e_{1}$ & $a$ & 0 & 0 \\
$a$ & $a$ & $\alpha e_{1}$ & 0 & 0 \\
$e_{2}$ & 0 & 0 & $e_{2}$ & 0 \\
$e_{3}$ & 0 & 0 & 0 & $e_{3}$
\end{tabular}

Thus $A \cong F[\sqrt{\alpha}] \oplus F \oplus F$. This is (3) in Theorem 2.1.

\subsection{A Is Not $\ell$-Reduced}

In this case, $a$ must be nilpotent. If $a^{2} \neq 0$, then $a^{2}$ must be a positive linear combination of elements in $S$. Since each of $e_{1}, e_{2}, e_{3}$ is not nilpotent, we must have $a^{2}=\beta a$ for some $0<\beta \in F$. Then $a$ is nilpotent implying that $a^{2}=\beta a=0$, which is a contradiction. Thus we must have $a^{2}=0$. There are two cases that we need to consider.

(1) $a e_{1}=a$.

Then $a e_{2}=a e_{3}=0$, and the multiplication table for $S=\left\{e_{1}, e_{2}, e_{3}, a\right\}$ is given below:

\begin{tabular}{c|cccc} 
& $e_{1}$ & $a$ & $e_{2}$ & $e_{3}$ \\
\hline$e_{1}$ & $e_{1}$ & $a$ & 0 & 0 \\
$a$ & $a$ & 0 & 0 & 0 \\
$e_{2}$ & 0 & 0 & $e_{2}$ & 0 \\
$e_{3}$ & 0 & 0 & 0 & $e_{3}$
\end{tabular}

Thus $A \cong(F 1+F a) \oplus F \oplus F$, where $a^{2}=0$ and $1 a=a 1=a$. This is (4) in Theorem 2.1.

(2) $a e_{1}=0$.

Without loss of generality, we may assume that $a e_{2}=a$ and $a e_{3}=0$. Then we have the following multiplication table for elements in $S$ :

\begin{tabular}{c|cccc} 
& $e_{1}$ & $e_{2}$ & $a$ & $e_{3}$ \\
\hline$e_{1}$ & $e_{1}$ & 0 & $a$ & 0 \\
$e_{2}$ & 0 & $e_{2}$ & 0 & 0 \\
$a$ & 0 & $a$ & 0 & 0 \\
$e_{3}$ & 0 & 0 & 0 & $e_{3}$
\end{tabular}

Let $T_{2}(F)$ be the $2 \times 2$ upper triangular matrix algebra over $F$ with the entrywise lattice order. Then $T_{2}(F)$ becomes an $\ell$-algebra over $F$ with a $d$-basis $\left\{e_{11}, e_{22}, e_{12}\right\}$. The multiplication table for $\left\{e_{11}, e_{22}, e_{12}\right\}$ is given below:

\begin{tabular}{c|ccc} 
& $e_{11}$ & $e_{22}$ & $e_{12}$ \\
\hline$e_{11}$ & $e_{11}$ & 0 & $e_{12}$ \\
$e_{22}$ & 0 & $e_{22}$ & 0 \\
$e_{12}$ & 0 & $e_{12}$ & 0
\end{tabular}


Since $\left\{e_{1}, e_{2}, a\right\}$ and $\left\{e_{11}, e_{22}, e_{12}\right\}$ have the same multiplication tables, we have $A \cong$ $T_{2}(F) \oplus F$. This is (5) in Theorem 2.1.

\section{1 Is a Sum of Two Disjoint Basic Elements}

Let $A$ be a unital $\ell$-algebra with a $d$-basis $S=\left\{e_{1}, e_{2}, a, b\right\}$ such that $1=e_{1}+e_{2}$. By Theorem 1.1, $a e_{i}=a$ for some $i=1$ or 2 . Then $e_{i} \wedge b=0$ implies that $a \wedge a b=0$. Similarly $b \wedge a b=0$. Thus $a b=\alpha e_{1}+\beta e_{2}$ for some $\alpha, \beta \in F^{+}$. By a similar argument, $b a=\gamma e_{1}+\delta e_{2}$, for some $\gamma, \delta \in F^{+}$.

\subsection{A Is $\ell$-Reduced}

We may assume that $e_{1} a=a$ and $e_{2} a=0$. Since $A$ contains no nonzero positive nilpotent elements, we also have $a e_{1}=a$ and $a e_{2}=0$. Then $a b=\alpha e_{1}+\beta e_{2}$ implies that $a b=e_{1} a b=\alpha e_{1}$. Similarly, $b a=\gamma e_{1}$.

Since $A$ is $\ell$-reduced, $a$ is not a nilpotent element, so $a^{2}$ is basic by Theorem 1.1. Thus $a^{2}=\sigma s$ for some $s \in S$ and $0<\sigma \in F$. Since $e_{1} \wedge a=0$ and $e_{1} a=a, s \neq a$. If $s=e_{2}$, then $a^{3}=\sigma\left(a e_{2}\right)=0$, which is a contradiction. Thus $s=e_{1}$ or $s=b$. We consider these two cases separately.

(1) $a^{2}=\sigma e_{1}$.

Suppose that $a b \neq 0$. Since $a b=\alpha e_{1}, a^{2} b=\alpha a$, so $\sigma\left(e_{1} b\right)=\alpha a$, which is a contradiction. Thus $a b=0$, and hence $b a=0$ since $(b a)^{2}=0$. Since $0=b a^{2}=\sigma b e_{1}$, $b e_{1}=0$. Thus $b e_{2}=b$, and hence $e_{1} b=0$ and $e_{2} b=b$. Now $b$ is not nilpotent implying that $b^{2}$ is a basic element, so $b^{2}=\lambda s$ for some $s \in S$ and $0<\lambda \in F$. Since $e_{2} \wedge b=0$ and $e_{2} b=b, s \neq b$. If $b^{2}=\lambda e_{1}$ or $b^{2}=\lambda a$, then $\lambda a=0$ or $\lambda a^{2}=0$, which are contradictions. Thus we must have $b^{2}=\lambda e_{2}$. Then we have the following multiplication table for the elements of $S$ :

\begin{tabular}{c|cccc} 
& $e_{1}$ & $a$ & $e_{2}$ & $b$ \\
\hline$e_{1}$ & $e_{1}$ & $a$ & 0 & 0 \\
$a$ & $a$ & $\sigma e_{1}$ & 0 & 0 \\
$e_{2}$ & 0 & 0 & $e_{2}$ & $b$ \\
$b$ & 0 & 0 & 0 & $\lambda e_{2}$
\end{tabular}

Depending on $\sqrt{\sigma} \in F$ or $\sqrt{\sigma} \notin F$ and $\sqrt{\lambda} \in F$ or $\sqrt{\lambda} \notin F$, we have the following cases:

$$
A \cong F[G] \oplus F[G], \quad A \cong F[G] \oplus F[\sqrt{\sigma}], \quad \text { or } \quad A \cong F[\sqrt{\sigma}] \oplus F[\sqrt{\lambda}],
$$

where $G$ is a group of order two. These are (6), (7), and (8) in Theorem 2.1.

(2) $a^{2}=\sigma b$.

Since $a^{2}=\sigma b$ with $0<\sigma \in F, T=\left\{e_{1}, e_{2}, a, a^{2}\right\}$ is also a $d$-basis for the $\ell$-algebra $A$ 
over $F$ and $a^{3}=\sigma(a b)=(\alpha \sigma) e_{1}$. Let $\mu=\alpha \sigma$. Then $\mu>0$ since $a^{3} \neq 0$, so we have the following multiplication table for elements in $T$ :

\begin{tabular}{c|cccc} 
& $e_{1}$ & $a$ & $a^{2}$ & $e_{2}$ \\
\hline$e_{1}$ & $e_{1}$ & $a$ & $a^{2}$ & 0 \\
$a$ & $a$ & $a^{2}$ & $\mu e_{1}$ & 0 \\
$a^{2}$ & $a^{2}$ & $\mu e_{1}$ & $\mu a$ & $b$ \\
$e_{2}$ & 0 & 0 & 0 & $e_{2}$
\end{tabular}

Now it is clear that if $\sqrt[3]{\mu} \in F$, then $A \cong F[G] \oplus F$, where $G$ is a group of order three, and if $\sqrt[3]{\mu} \notin F$, then $A \cong F[\sqrt[3]{\mu}] \oplus F$, where $F[\sqrt[3]{\mu}]$ is the $\ell$-field extension of $F$ of order three. Those are (9) and (10) in Theorem 2.1.

\subsection{A Is Not $\ell$-Reduced}

Since $A$ is not $\ell$-reduced, $a$ or $b$ is nilpotent. Let's assume that $e_{1} a=a$, and hence $e_{2} a=0$. Suppose that $a$ is nilpotent and $a^{2} \neq 0$. Then

$$
a^{2}=\alpha_{1} e_{1}+\alpha_{2} e_{2}+\alpha_{3} a+\alpha_{4} b, \quad \alpha_{i} \in F^{+}
$$

Let $k$ be the smallest positive integer such that $a^{k}=0$. Then $k \geq 3$ and

$$
0=a^{k}=a^{2} a^{k-2}=\alpha_{1} e_{1} a^{k-2}+\alpha_{2} e_{2} a^{k-2}+\alpha_{3} a^{k-1}+\alpha_{4} b a^{k-2},
$$

so $\alpha_{1} a^{k-2}=\alpha_{3} a^{k-1}=0$. Thus $\alpha_{1}=\alpha_{3}=0$, and hence $a^{2}=\alpha_{2} e_{2}+\alpha_{4} b$. Then $a^{2}=e_{1} a^{2}=\alpha_{4}\left(e_{1} b\right)$, which implies that $\alpha_{2}=0$ and $a^{2}=\alpha_{4} b$. Then $a^{2} \neq 0$ implies that $\alpha_{4}>0$. So $\left\{e_{1}, e_{2}, a, a^{2}\right\}$ is a $d$-basis of $A$ over $F$. Similarly if $b$ is nilpotent and $b^{2} \neq 0$, then $\left\{e_{1}, e_{2}, b, b^{2}\right\}$ is a $d$-basis of $A$ over $F$. Therefore we only need to consider the following cases.

(i) $a^{2}=b^{2}=0$.

(ii) $a$ is nilpotent, but $a^{2} \neq 0$. By the above argument, $a^{2}=\alpha_{4} b$ for some $0<\alpha_{4} \in F$. Thus $T=\left\{e_{1}, e_{2}, a, a^{2}\right\}$ is also a $d$-basis for $A$ over $F$.

(iii) $a^{2}=0$ and $b$ is not nilpotent.

Below, we consider each case in detail.

4.2.1. $a^{2}=b^{2}=0$

From $b a=\gamma e_{1}+\delta e_{2}$, we have $0=b a^{2}=\gamma e_{1} a+\delta e_{2} a=\gamma e_{1} a$, so $\gamma=0$, and hence $b a=\delta e_{2}$.

(1) $\delta>0$. Then $b a=\delta e_{2} \neq 0$ implies that $e_{2} b=b, e_{1} b=0, a e_{2}=a$, and $a e_{1}=0$. Then $a b=\alpha e_{1}+\beta e_{2}$ implies that $0=a^{2} b=\alpha a e_{1}+\beta a e_{2}=\beta a$, so $\beta=0$ and $a b=\alpha e_{1}$. Thus $a b a=\alpha e_{1} a=\delta a e_{2}$ implies that $\alpha=\delta$. Since $a b=\alpha e_{1} \neq 0, b e_{1}=b$ and $b e_{2}=0$. 
Let $a^{\prime}=a / \alpha$. Then $\left\{e_{1}, e_{2}, a^{\prime}, b\right\}$ is also a $d$-basis of $A$ with the following multiplication table:

\begin{tabular}{c|cccc} 
& $e_{1}$ & $e_{2}$ & $a^{\prime}$ & $e_{2}$ \\
\hline$e_{1}$ & $e_{1}$ & $a$ & $a^{\prime}$ & 0 \\
$e_{2}$ & 0 & $e_{2}$ & 0 & $b$ \\
$a^{\prime}$ & 0 & $a^{\prime}$ & 0 & $e_{1}$ \\
$b$ & $b$ & 0 & $e_{2}$ & 0
\end{tabular}

It is clear now that $A \cong M_{2}(F)$, the $2 \times 2$ matrix $\ell$-algebra over $F$. This is (11) in Theorem 2.1.

(2) $\delta=0$. Then $b a=0$. From $a b=\alpha e_{1}+\beta e_{2}$, we have $0=a(b a)=(a b) a=\alpha e_{1} a+\beta e_{2} a=$ $\alpha a$, so $\alpha=0$. Thus $a b=\beta e_{2}$, and hence $0=e_{2} a b=\beta e_{2}$, which implies that $\beta=0$, so $a b=0$. Therefore, $F a+F b$ is a zero $\ell$-ring. There are the following cases.

(2a) If $a e_{1}=a, e_{1} b=b$, and $b e_{1}=b$, then $A \cong(F 1+F a+F b) \oplus F$, where $F a+F b$ is the zero $\ell$-ring.

(2b) If $a e_{1}=a, e_{2} b=b$, and $b e_{2}=b$, then $A \cong(F 1+F a) \oplus(F 1+F b)$, where $a^{2}=b^{2}=0$. If $a e_{2}=a$, then the multiplication table for $\left\{e_{1}, e_{2}, a\right\}$ is given below:

\begin{tabular}{c|ccc} 
& $e_{1}$ & $e_{2}$ & $a$ \\
\hline$e_{1}$ & $e_{1}$ & 0 & $a$ \\
$e_{2}$ & 0 & $e_{2}$ & 0 \\
$a$ & 0 & $a$ & 0
\end{tabular}

Clearly $F e_{1}+F e_{2}+F a$ is isomorphic to $T_{2}(F)$, and hence there are the following cases depending on the products between $e_{1}, e_{2}$, and $b$.

(2c) If $e_{1} b=b$ and $b e_{1}=b$, then $A \cong T_{2}(F)+F b$ with $e_{11} b=b e_{11}=b, b e_{12}=0$, and $b^{2}=0$.

(2d) If $e_{1} b=b$ and $b e_{2}=b$, then $A \cong T_{2}(F)+F b$ with $e_{11} b=b, b e_{22}=b$, and $b^{2}=0$.

(2e) If $e_{2} b=b$ and $b e_{1}=b$, then $A \cong T_{2}(F)+F b$ with $e_{22} b=b, b e_{11}=b, e_{12} b=b e_{12}=$ 0 , and $b^{2}=0$.

(2f) If $e_{2} b=b$ and $b e_{2}=b$, then $A \cong T_{2}(F)+F b$ with $e_{22} b=b e_{22}=b, e_{12} b=0$, and $b^{2}=0$.

Two more cases we need to consider are (i) $a e_{1}=a, e_{1} b=b, b e_{1}=0$ and (ii) $a e_{1}=a$, $e_{2} b=b, b e_{2}=0$. But when we switch $a$ and $b$, the case (i) becomes (2c), and when we switch $a$ and $b$, and also switch $e_{1}$ and $e_{2}$, the case (ii) becomes (2f). Thus every possible case when $\delta=0$ is covered. The above discussion has produced (12)-(17) in Theorem 2.1.

\subsection{2. $a$ Is Nilpotent and $a^{2} \neq 0$}

As we have noticed before, in this case $T=\left\{e_{1}, e_{2}, a, a^{2}\right\}$ is a $d$-basis for the $\ell$-algebra $A$ over $F$. Since $a \wedge e_{1}=0$ implies that $a^{3} \wedge a^{2}=0$ and $a^{2} \wedge e_{1}=0$ implies that $a^{3} \wedge a=0, a^{3}=\beta_{1} e_{1}$, for some $\beta_{1} \in F^{+}$. If $\beta_{1} \neq 0$, then $a$ cannot be nilpotent, so we must have $\beta_{1}=0$. Therefore $a^{3}=0$, and $A \cong\left(F 1+F a+F a^{2}\right) \oplus F$ with $a^{3}=0$. This is (18) in Theorem 2.1. 


\subsection{3. $a^{2}=0$ and $b$ Is Not Nilpotent}

Since $b$ is not nilpotent, $b^{2}$ is basic, so $b^{2}=\epsilon s$ for some $s \in S$ and $0<\epsilon \in F$. Clearly $s \neq a$ or $b$. Thus either $b^{2}=\epsilon e_{1}$ or $b^{2}=\epsilon e_{2}$. In the first case, we have $b^{2} a=\epsilon e_{1} a=b\left(\gamma e_{1}+\delta e_{2}\right)=\gamma b e_{1}$, so $\epsilon a=\gamma b$, which is a contradiction. Thus we must have $b^{2}=\epsilon e_{2}$. If $a e_{2}=a$, then $a b^{2}=\epsilon a$, so $\left(\alpha e_{1}+\beta e_{2}\right) b=\epsilon a$, which implies that $\beta b=\epsilon a$, a contradiction. Thus $a e_{1}=a$, and hence $a e_{2}=0$. Then $a b=b a=0$. Therefore we have the following multiplication table for the elements of $S$ :

\begin{tabular}{c|cccc} 
& $e_{1}$ & $a$ & $e_{2}$ & $b$ \\
\hline$e_{1}$ & $e_{1}$ & $a$ & 0 & 0 \\
$a$ & $a$ & 0 & 0 & 0 \\
$e_{2}$ & 0 & 0 & $e_{2}$ & $b$ \\
$b$ & 0 & 0 & $b$ & $\epsilon e_{2}$
\end{tabular}

From the above table, it is clear that if $\sqrt{\epsilon} \in F$, then $A \cong F[G] \oplus(F 1+F a)$ with $a^{2}=0$, where $G$ is a group of order two, and if $\sqrt{\epsilon} \notin F$, then $A \cong F[\sqrt{\epsilon}] \oplus(F 1+F a)$ with $a^{2}=0$, where $F[\sqrt{\epsilon}]$ is a quadratic extension $\ell$-field of $F$. Those are (19) and (20) in Theorem 2.1.

Now we have constructed all lattice orders on $A$ such that 1 is a sum of two disjoint basic elements.

\section{1 Is a Basic Element}

In this section, $A$ has a $d$-basis $S=\{1, a, b, c\}$. We still first consider the $\ell$-reduced case.

\subsection{A Is $\ell$-Reduced}

Since $a$ is not nilpotent, by Theorem 1.1, there exist positive integer $n_{a}$ and $0<\alpha_{a} \in F$ such that $a^{n_{a}}=\alpha_{a}$ 1. We also assume that $n_{a}$ is the smallest positive integer such that $a^{n_{a}}=\alpha_{a} 1$. Similarly $b^{n_{b}}=\alpha_{b} 1$ and $c^{n_{c}}=\alpha_{c} 1$, for some positive integers $n_{b}, n_{c}$, and $0<\alpha_{b}, \alpha_{c} \in F$. By Theorem 1.1 again, each $a^{k}$ is a basic element for $k \geq 1$.

Lemma 5.1. The subset $\left\{1, a, a^{2}, \ldots, a^{n_{a}-1}\right\}$ of $A$ is a disjoint set of $A$.

Proof. Let $1 \leq i<n_{a}$. Since $a^{i}$ is a basic element, $a^{i}=\alpha$ s for some $s \in S$ and $0<\alpha \in F$. Since $i<n_{a}, s \neq 1$, so $1 \wedge s=0$ implies that $1 \wedge a^{i}=0$. Now let $0 \leq u<v<n_{a}$, then $a^{u} \wedge a^{v}=a^{u}\left(1 \wedge a^{v-u}\right)=0$ by the above argument.

Since $A$ is four-dimensional, $n_{a} \leq 4$ by Lemma 5.1. We claim that $n_{a} \neq 3$. Supposing that $n_{a}=3$, then we may assume that $\left\{1, a, a^{2}, b\right\}$ is a $d$-basis. By Theorem $1.1, a b$ is a basic element and $a b \wedge a=a(b \wedge 1)=0, a b \wedge b=(a \wedge 1) b=0, a b \wedge a^{2}=a(b \wedge a)=0$, so $a b=\alpha 1$ for some $0<\alpha \in F$. Thus $a^{3} b=\alpha a^{2}$, and hence $\alpha_{a} b=\alpha a^{2}$, which is a contradiction. Hence $n_{a} \neq 3$, so $n_{a}=4$ or $n_{a}=2$.

By the above analysis, we need to consider two cases: (i) one of the following: $n_{a}, n_{b}$ $n_{c}$, is 4 , and we may assume that $n_{a}=4$; (ii) $n_{a}=n_{b}=n_{c}=2$. 


\subsection{1. $n_{a}=4$ and Hence $\left\{1, a, a^{2}, a^{3}\right\}$ Is a Disjoint Subset of Basic Elements of $A$}

In this case $T=\left\{1, a, a^{2}, a^{3}\right\}$ is a $d$-basis of $A$ over $F$, and we have the following multiplication table for the elements in $T$ :

\begin{tabular}{c|cccc} 
& 1 & $a$ & $a^{2}$ & $a^{3}$ \\
\hline 1 & 1 & $a$ & $a^{2}$ & $a^{3}$ \\
$a$ & $a$ & $a^{2}$ & $a^{3}$ & $\alpha_{a}$ \\
$a^{2}$ & $a^{2}$ & $a^{3}$ & $\alpha_{a} 1$ & $\alpha_{a} a$ \\
$a^{3}$ & $a^{3}$ & $\alpha_{a} 1$ & $\alpha_{a} a$ & $\alpha_{a} a^{2}$
\end{tabular}

We have the following three cases to be considered.

(1) If $\sqrt[4]{\alpha_{a}} \in F$, then $A \cong F[G]$, where $G$ is a cyclic group of order four.

(2) If the polynomial $f(x)=x^{4}-\alpha_{a} \in F[x]$ is irreducible, then $A \cong F\left[\sqrt[4]{\alpha_{a}}\right]$, which is an $\ell$-field extension of $F$ of order four.

(3) The polynomial $f(x)=x^{4}-\alpha_{a} \in F[G]$ is not irreducible and $\sqrt[4]{\alpha_{a}} \notin F$.

Suppose that $x^{4}-\alpha_{a}=\left(x^{2}+a_{1} x+b_{1}\right)\left(x^{2}+a_{2} x+b_{2}\right)$. Then we have $a_{1}+a_{2}=0, b_{1}+b_{2}+$ $a_{1} a_{2}=0, b_{1} a_{2}+b_{2} a_{1}=0$, and $b_{1} b_{2}=-\alpha_{a}<0$. Thus $a_{2}=-a_{1}$ and $0=b_{1} a_{2}+b_{2} a_{1}=a_{1}\left(-b_{1}+b_{2}\right)$. If $a_{1} \neq 0$, then $-b_{1}+b_{2}=0$, and hence $b_{1}=b_{2}$, which implies that $b_{1} b_{2}=b_{1}^{2}=-\alpha_{a}<0$, a contradiction. So we must have $a_{1}=0$, and hence $a_{2}=0$. Then $b_{1}+b_{2}=0$ and $b_{1} b_{2}=-\alpha_{a}$ implies that $b_{2}=-b_{1}$ and $b_{1}^{2}=b_{2}^{2}=\alpha_{a}$. We may assume that $b_{1}>0$. It follows from $\sqrt[4]{\alpha_{a}} \notin F$ that $\sqrt{b_{1}} \notin F$. Let $c=a^{2} / b_{1}$. Then $V=\{1, c, a, a c\}$ is also a $d$-basis of $A$ over $F$ and the multiplication table for the elements in $V$ is given below:

\begin{tabular}{c|cccc} 
& 1 & $c$ & $a$ & $a c$ \\
\hline 1 & 1 & $c$ & $a$ & $a c$ \\
$c$ & $c$ & 1 & $a c$ & $a$ \\
$a$ & $a$ & $a c$ & $b_{1} c$ & $b_{1} 1$ \\
$a c$ & $a c$ & $a$ & $b_{1} 1$ & $b_{1} c$
\end{tabular}

Then $A \cong F[G]+F[G] a$, where $G=\{e, c\}$ is a group of order two and $a^{2}=\alpha c, 0<\alpha \in F$, and $\sqrt{\alpha} \notin F$. These are (21), (22), and (23) in Theorem 2.1.

\subsection{2. $n_{a}=n_{b}=n_{c}=2$}

Then $S=\{1, a, b, c\}$ is a $d$-basis and $a^{2}=\alpha_{a} 1, b^{2}=\alpha_{b} 1$, and $c^{2}=\alpha_{c} 1$. Since $a b \wedge a=a b \wedge b=0$ and $a b$ is basic, $a b=\alpha 1$ or $a b=\alpha c$ for some $0<\alpha \in F$. In the first case, $a b^{2}=\alpha b$ implies that $\alpha_{b} a=\alpha b$, which is a contradiction. Thus $a b=\alpha c$ for some $0<\alpha \in F$. Then we may replace $c$ by $a b$ to get a $d$-basis $T=\{1, a, b, a b\}$.

Since $b a \wedge a=b a \wedge b=0$ and $b a$ is basic, $b a=\beta 1$ or $b a=\beta(a b)$ for some $0<\beta \in F$. If $b a=\beta 1$, then $\alpha_{a} b=\beta a$, which is a contradiction. Thus $b a=\beta(a b)$, for some $0<\beta \in F$, and hence

$$
\alpha_{b} \alpha_{a} 1=b(b a) a=\beta b(a b) a=\beta^{2}(a b)(b a)=\beta^{2} \alpha_{b} \alpha_{a} 1 .
$$


So $\beta=1$ and the multiplication table for the elements of $T$ is given below:

\begin{tabular}{c|cccc} 
& 1 & $a$ & $b$ & $a b$ \\
\hline 1 & 1 & $a$ & $b$ & $a b$ \\
$a$ & $a$ & $\alpha_{a} 1$ & $a b$ & $\alpha_{a} b$ \\
$b$ & $b$ & $a b$ & $\alpha_{b} 1$ & $\alpha_{b} a$ \\
$a b$ & $a b$ & $\alpha_{a} b$ & $\alpha_{b} a$ & $\alpha_{a} \alpha_{b} 1$
\end{tabular}

There are the following cases.

(1) $\sqrt{\alpha_{a}}$ and $\sqrt{\alpha_{b}} \in F$.

Replacing $a$ and $b$ by $a / \sqrt{\alpha_{a}}$ and $b / \sqrt{\alpha_{b}}$, respectively, we have $a^{2}=b^{2}=1$. Therefore $A \cong F[G]$, where $G$ is the Klein four group.

(2) $\sqrt{\alpha_{a}} \notin F$ and $\sqrt{\alpha_{b}} \in F$ (or $\sqrt{\alpha_{a}} \in F$ and $\sqrt{\alpha_{b}} \notin F$ ).

Replacing $b$ by $b / \sqrt{\alpha_{b}}$, we may assume that $b^{2}=1$. Therefore $A \cong F\left[\sqrt{\alpha_{a}}\right][G]$, where $F\left[\sqrt{\alpha_{a}}\right]$ is the quadratic extension $\ell$-field of $F$ and $G$ is a group of order two.

(3) $\sqrt{\alpha_{a}} \notin F$ and $\sqrt{\alpha_{b}} \notin F$.

If $\sqrt{\alpha_{b}} \in F\left[\sqrt{\alpha_{a}}\right]$, then $A \cong F\left[\sqrt{\alpha_{a}}\right][G]$, where $G$ is a group of order two. We omit the checking and leave it to the reader.

If $\sqrt{\alpha_{b}} \notin F\left[\sqrt{\alpha_{a}}\right]$, then $A \cong F\left[\sqrt{\alpha_{a}}\right]\left[\sqrt{\alpha_{b}}\right]$, where $F\left[\sqrt{\alpha_{a}}\right]$ is a quadratic extension $\ell$-field of $F$ and $F\left[\sqrt{\alpha_{a}}\right]\left[\sqrt{\alpha_{b}}\right]$ is a quadratic extension $\ell$-field of $F\left[\sqrt{\alpha_{a}}\right]$. Those are (24), (25), and (26) in Theorem 2.1.

\subsection{A Is Not $\ell$-Reduced}

Let $S=\{1, a, b, c\}$ be a $d$-basis of $A$ over $F$. Then some elements of $a, b, c$ must be nilpotent. We first observe the following result.

Lemma 5.2. Let $x, y \in\{1, a, b, c\}$. If either $x$ or $y$ is nilpotent and the other one is not nilpotent, then $x y$ is nilpotent.

Proof. Let $x$ be nilpotent and let $y$ be not. By Theorem 1.1, there exist a positive integer $n_{y}$ and $0<\alpha_{y} \in F$ such that $y^{n_{y}}=\alpha_{y} 1$. If $x y=0$, then $x y^{n_{y}}=0$ implies that $\alpha_{y} x=0$, which is a contradiction. Thus $x y \neq 0$, so $x y$ is basic by Theorem 1.1. If $x y$ is not nilpotent, then $x y=\gamma z$ for some $0<\gamma \in F$ and $z \in\{1, a, b, c\}$ and $z$ is not nilpotent. By Theorem 1.1, there exist a positive integer $n_{z}, 0<\alpha_{z} \in F$ such that $z^{n_{z}}=\alpha_{z} 1$. Let $n>1$ be the smallest positive integer such that $x^{n}=0$. Then $0=x^{n} y=x^{n-1}(x y)=\gamma x^{n-1} z$, and hence $0=\gamma x^{n-1} z$ implies that $0=\gamma x^{n-1} z^{n_{z}}=\alpha_{z} \gamma x^{n-1}$. Thus $x^{n-1}=0$, which is a contradiction. Therefore $x y$ is nilpotent.

By Lemma 5.2, $\{a, b, c\}$ cannot have exactly one nilpotent element. Suppose not, let $a$ be the unique nilpotent element. Since $a b \wedge a=0, a b$ cannot be a positive scalar multiple of $a$, so $a b$ cannot be a nilpotent element, which is a contradiction by Lemma 5.2. So there are following two cases. 


\subsection{1. $a, b$, and c Are All Nilpotent}

(1) Either $a, b$, or $c$ has nonzero square.

We may assume that $a^{2} \neq 0$. Since $a^{2} \wedge a=a(a \wedge 1)=0, a^{2}=\alpha 1+\beta b+\gamma c$, where $\alpha, \beta, \gamma \in F^{+}$. Let $k$ be the smallest positive integer such that $a^{k}=0$. Then $0=a^{k}=$ $a^{k-2} a^{2}=\alpha a^{k-2}+\beta a^{k-2} b+\gamma a^{k-2} c$ implies $\alpha a^{k-2}=0$, so $\alpha=0$. Thus $a^{2}=\beta b+\gamma c$.

(1a) $\beta>0$ and $\gamma>0$.

Since $a^{2} \wedge a b=a(a \wedge b)=0, b \wedge a b=c \wedge a b=0$. Also $a \wedge a b=a(1 \wedge b)=0$.

Thus $a b=\delta 1$. Then we have $0=a^{k} b=\delta a^{k-1}$, so $\delta=0$ and $a b=0$. Similarly $b a=0, a c=0$, and $c a=0$. Since $a^{2}=\beta b+\gamma c, 0=a^{2} b=\beta b^{2}+\gamma c b$ implies that $b^{2}=c b=0$. Similarly $c^{2}=b c=0$.

Replacing $b$ and $c$ by $\beta b$ and $\gamma c$, respectively, we may assume that $a^{2}=b+c$. Therefore we have the following multiplication table:

\begin{tabular}{c|cccc} 
& 1 & $a$ & $b$ & $c$ \\
\hline 1 & 1 & $a$ & $b$ & $c$ \\
$a$ & $a$ & $b+c$ & 0 & 0 \\
$b$ & $b$ & 0 & 0 & 0 \\
$c$ & $c$ & 0 & 0 & 0
\end{tabular}

Then $A \cong F 1+F a+F b+F c$, where $a^{2}=b+c$ and the product of any other two elements of $a, b, c$ is zero. This is (27) in Theorem 2.1. We want to point out that this case provides an example in which the product of two basic elements may not be basic.

(1b) $\beta=0$ or $\gamma=0$.

We may assume that $\gamma=0$, so $\beta>0$. Then $a^{2}=\beta b$, and $a(a a)=(a a) a$ implies that $\beta(a b)=\beta(b a)$, so $a b=b a$.

(i) $a b=b a \neq 0$.

Then $a b=\delta c$ for some $0<\delta \in F$. Thus $a^{3}=a a^{2}=\beta(a b)=\beta \delta c$, and hence we may replace $b$ by $a^{2}$ and $c$ by $a^{3}$ to get another $d$-basis $T=\left\{1, a, a^{2}, a^{3}\right\}$. Therefore $A \cong F 1+F a+F a^{2}+F a^{3}$ with $a^{4}=0$. This is (28) in Theorem 2.1.

(ii) $a b=b a=0$.

Then $\beta b^{2}=a^{2} b=0$, so $b^{2}=0$. Since $a c=\mu b$ for some $0 \leq \mu \in F, a^{2} \wedge a c=0$ and $a^{2}=\beta b$ implies that $\mu=0$, so $a c=0$. Similarly $c a=0.0=a^{2} c=\beta(b c)$, so $b c=0$. Similarly, $c b=0$. Now replacing $b$ by $a^{2}$, we obtain another $d$-basis $T=\left\{1, a, a^{2}, c\right\}$.

If $c^{2} \neq 0$, then $c^{2} \wedge c=c^{2} \wedge 1=0$ implies that $c^{2}=\alpha_{1} a+\alpha_{2} a^{2}$. Then it follows from $a c=0$ and $a^{3}=0$ that $\alpha_{1} a^{2}=0$, so $\alpha_{1}=0$. Thus $c^{2}=\alpha_{2} a^{2}$ with $0<\alpha_{2} \in F$. Hence $A \cong F 1+F a+F a^{2}+F c$ with $c^{2}=\alpha a^{2}$ for some $0<\alpha \in F$ and $x y=0$ for any two elements $x$ and $y$ in $\left\{a, a^{2}, c\right\}$ not both $a$ or $c$. This is (29) in Theorem 2.1.

If $c^{2}=0$, then $A \cong F 1+F a+F a^{2}+F c$ with $x y=0$ for any two elements $x$ and $y$ in $\left\{a, a^{2}, c\right\}$ which are not both $a$. This is (30) in Theorem 2.1.

We provide some familiar examples for the above two cases. Consider $4 \times 4$ matrix algebra over $F$. Let $1=e_{11}+e_{22}+e_{33}+e_{44}, a=e_{12}+e_{23}$, and $c=e_{14}+2 e_{43}$. Then $a^{2}=e_{13}$ and $c^{2}=2 a^{2}$. Clearly $F 1+F a+F a^{2}+F c$ is a four-dimensional 
$\ell$-algebra over $F$ with coordinatewise order. If $c=e_{13}+e_{43}$, then $c^{2}=0$ and $F 1+F a+F a^{2}+F c$ is a four-dimensional $\ell$-algebra over $F$ with $c^{2}=0$.

(2) $a^{2}=b^{2}=c^{2}=0$.

(2a) $x y=0$ for any two elements in $\{a, b, c\}$.

Then $A \cong F 1+F a+F b+F c$, where $F a+F b+F c$ is zero ring. This is (31) in Theorem 2.1.

(2b) At least one product of two different elements in $\{a, b, c\}$ is nonzero.

Without loss of generality, we may assume that $a b \neq 0$. Then $a b=\alpha c$ for some $0<\alpha \in F$, and we may replace $c$ by $a b$ to form another $d$-basis $T=\{1, a, b, a b\}$. If $b a=0$, then we have the following multiplication table:

\begin{tabular}{c|cccc} 
& 1 & $a$ & $b$ & $a b$ \\
\hline 1 & 1 & $a$ & $b$ & $a b$ \\
$a$ & $a$ & 0 & $a b$ & 0 \\
$b$ & $b$ & 0 & 0 & 0 \\
$a b$ & $a b$ & 0 & 0 & 0
\end{tabular}

Thus $A \cong F 1+F a+F b+F(a b)$ with $a^{2}=b^{2}=b a=0$. This is (32) in Theorem 2.1. If $b a \neq 0$, then $b a=\beta(a b)$ for some $0<\beta \in F$ and we have the following multiplication table:

\begin{tabular}{c|cccc} 
& 1 & $a$ & $b$ & $a b$ \\
\hline 1 & 1 & $a$ & $b$ & $a b$ \\
$a$ & $a$ & 0 & $a b$ & 0 \\
$b$ & $b$ & $\beta(a b)$ & 0 & 0 \\
$a b$ & $a b$ & 0 & 0 & 0
\end{tabular}

Thus $A \cong F 1+F a+F b+F(a b)$ with $a^{2}=b^{2}=0$ and $b a=\beta(a b)$ for some $0<\beta \in F$. This is (33) in Theorem 2.1.

\subsubsection{Two Elements in $\{a, b, c\}$ Are Nilpotent}

We may assume that $a$ and $b$ are nilpotent elements. Then we must have $c^{2}=\alpha 1$ for some $0<\alpha \in F$. By Lemma 5.2, $a c=\beta b$ for some $0<\beta \in F$. If $a^{2} \neq 0$, then $a^{2} \wedge a=a(a \wedge 1)=0$ and that 1 and $c$ are not nilpotent implies that $a^{2}=\gamma b$ for some $0<\gamma \in F$. Thus $a c \wedge a^{2} \neq 0$, which is a contradiction, so $a^{2}=0$. Similarly, $b^{2}=0$. Replacing $a$ by $a / \beta$ and then replacing $b$ by $a c$, we have another $d$-basis $\{1, c, a, a c\}$ with the following multiplication table:

\begin{tabular}{c|cccc} 
& 1 & $c$ & $a$ & $a c$ \\
\hline 1 & 1 & $c$ & $a$ & $a c$ \\
$c$ & $c$ & $\alpha 1$ & $a c$ & $\alpha a$ \\
$a$ & $a$ & $a c$ & 0 & 0 \\
$a c$ & $a c$ & $\alpha a$ & 0 & 0
\end{tabular}


If $\sqrt{\alpha} \in F$, then replacing $c$ by $c / \sqrt{\alpha}$, we have that $A \cong F[G]+F[G] a$ is a commutative $\ell$-algebra, where $G$ is a group of order two and $a^{2}=0$.

If $\sqrt{\alpha} \notin F$, then $A \cong F[\sqrt{\alpha}]+F[\sqrt{\alpha}] a$ is a commutative $\ell$-algebra over $F$, where $F[\sqrt{\alpha}]$ is a quadratic extension $\ell$-field of $F$ and $a^{2}=0$. Those are (34) and (35) in Theorem 2.1.

This completes the proof of Theorem 2.1.

\section{An Application}

For an $\ell$-ring $R$, its $\ell$-radical $\ell-N(R)$ is defined as

$$
\ell-N(R)=\left\{a \in R: \exists n=n(a) \text { such that } x_{0}|a| x_{1}|a| \cdots|a| x_{n}=0, \forall x_{0}, \ldots, x_{n} \in R\right\},
$$

(see [1, Definition, page 45]). $\ell-N(R)$ is the sum of all of the nilpotent $\ell$-ideals of $R$ [1, Theorem 5]. It is clear that for an $\ell$-algebra $A, \ell-N(A)$ is an $\ell$-ideal of $A$.

A positive derivation on an $\ell$-ring $R$ is a function $\delta: R \rightarrow R$ such that for any $a, b \in R$, $\delta(a+b)=\delta(a)+\delta(b), \delta(a b)=a \delta(b)+\delta(a) b$, and $\delta\left(R^{+}\right) \subseteq R^{+}$. For an Archimedean $f$-ring $R$, if $\delta$ is a positive derivation on $R$, then $\delta(R) \subseteq \ell-N(R)[2,3]$. Although we believe this result is true for a unital $\ell$-algebra with a $d$-basis, we lack ability to prove it in general. In the following, we prove this result for unital four-dimensional $\ell$-algebras with a $d$-basis based on the classifications obtained in Theorem 2.1.

Theorem 6.1. Let $A$ be a unital four-dimensional $\ell$-algebra with a d-basis. If $\delta$ is a positive derivation on $A$, then $\delta(A) \subseteq l-N(A)$.

Proof. We notice first that since $1>0$, for any $a \in A$ and $\alpha \in F, \delta(\alpha a)=\alpha \delta(a)$ [4]; that is, $\delta$ is actually a linear transformation of $A$. Let $1=e_{1}+\cdots+e_{n}$, where $e_{1}, \ldots, e_{n}$ are distinct elements from the $d$-basis and $n \leq 4$. Since $\delta(1)=\delta(1)+\delta(1)$ implies $\delta(1)=0,0=\delta\left(e_{1}\right)+\cdots+\delta\left(e_{n}\right)$. Then $\delta$ is positive implying that $\delta\left(e_{i}\right)=0$ for each $i=1, \ldots, n$. Let $a$ be an element in the $d$-basis, by Theorem 1.1, either $a$ is nilpotent or $a^{n_{a}}=\alpha_{a} e_{i}$ for some $n_{a} \geq 1,0<\alpha_{a} \in F$, and $e_{i}$. If $a$ is nilpotent, then $\delta(a)$ is also nilpotent [4]. If $a^{n_{a}}=\alpha_{a} e_{i}$, then $\delta\left(a^{n_{a}}\right)=\delta\left(\alpha_{a} e_{i}\right)=\alpha_{a} \delta\left(e_{i}\right)=0$ and $\delta$ is positive also implying that $\delta(a)$ is nilpotent. Thus for each element $a$ in the $d$-basis, $\delta(a)$ is nilpotent, so if $A$ is $\ell$-reduced, then $\delta(a)=0$ for each $a$ in the $d$-basis. Therefore $\delta(A)=0$.

We only need to consider those cases in Theorem 2.1 in which $A$ is not $\ell$-reduced. In cases (4)-(5), (12)-(20), and (27)-(35), the $\ell$-radical consists of all nilpotent elements of the $\ell$-algebra $A$. From the argument in the previous paragraph, for each element $x$ in the $d$-basis, $\delta(x)$ is nilpotent, so $\delta(x) \in \ell-N(A)$. Thus $\delta(A) \subseteq \ell-N(A)$ for those cases.

The only case left is (11) in which $\ell-N\left(M_{2}(F)\right)=0$, but $M_{2}(F)$ contains nonzero nilpotent elements $e_{12}$ and $e_{21}$. We show that $\delta\left(e_{12}\right)=\delta\left(e_{21}\right)=0$. Then together with $\delta\left(e_{11}\right)=\delta\left(e_{22}\right)=0$, we have $\delta\left(M_{2}(F)\right)=0$. Since $\left(e_{12}+e_{21}\right)^{2}=1$,

$$
\left(e_{12}+e_{21}\right) \delta\left(e_{12}+e_{21}\right)+\delta\left(e_{12}+e_{21}\right)\left(e_{12}+e_{21}\right)=0,
$$

so $\delta$ is positive implying that $\left(e_{12}+e_{21}\right) \delta\left(e_{12}+e_{21}\right)=0$. Multiplying this last equation by $\left(e_{12}+e_{21}\right)$ from the left, we have $\delta\left(e_{12}+e_{21}\right)=0$. Hence $\delta\left(e_{12}\right)=\delta\left(e_{21}\right)=0$. Therefore $\delta\left(M_{2}(F)\right)=0$.

This completes the proof. 


\section{References}

[1] G. Birkhoff and R. S. Pierce, "Lattice-ordered rings," Anais da Academia Brasileira de Ciências, vol. 28, pp. 41-69, 1956.

[2] P. Colville, G. Davis, and K. Keimel, "Positive derivations on $f$-rings," Journal of the Australian Mathematical Society, vol. 23, no. 3, pp. 371-375, 1977.

[3] M. Henriksen and F. A. Smith, "Some properties of positive derivations on $f$-rings," in Ordered Fields and Real Algebraic Geometry, vol. 8 of Contemporary Mathematics, pp. 175-184, American Mathematical Society, Providence, RI, USA, 1982.

[4] J. Ma and R. H. Redfield, "Positive derivations on Archimedean lattice-ordered rings," Positivity, vol. 13, no. 1, pp. 165-191, 2009.

[5] J. Ma, "Finite-dimensional lattice-ordered algebras with d-elements," Journal of Algebra, vol. 280, no. 1, pp. 232-243, 2004.

[6] J. Ma, "Lattice-ordered algebras with a d-basis," Journal of Algebra, vol. 299, no. 2, pp. 731-746, 2006. 


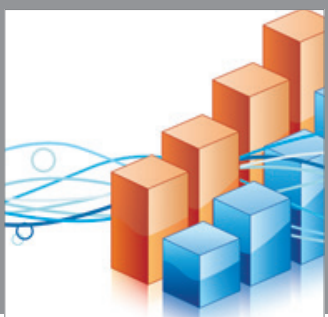

Advances in

Operations Research

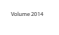

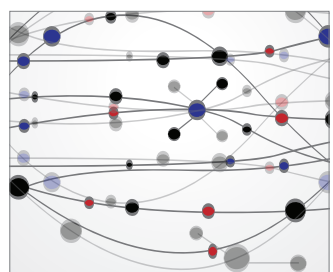

\section{The Scientific} World Journal
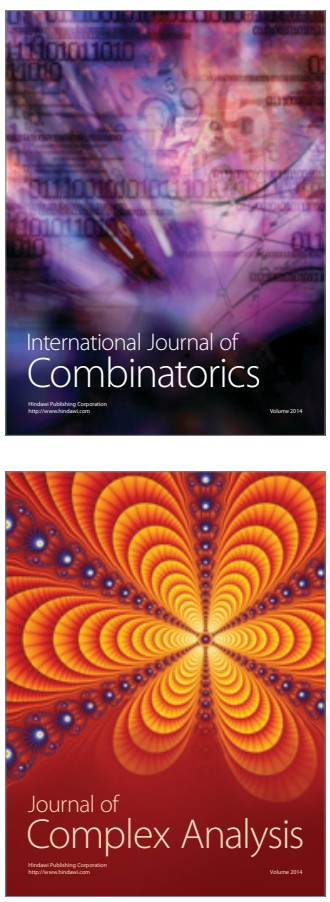

International Journal of

Mathematics and

Mathematical

Sciences
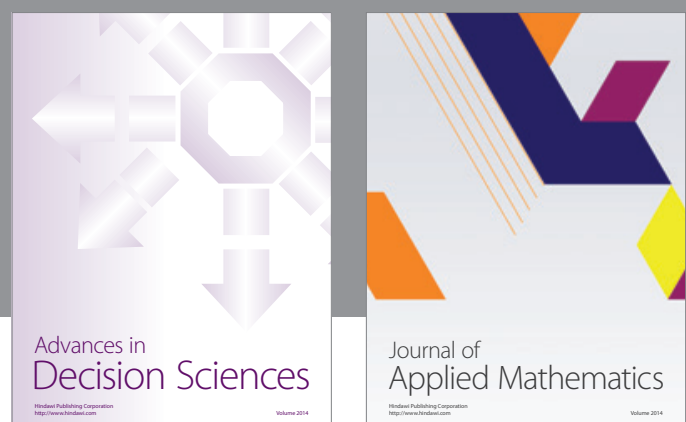

Journal of

Applied Mathematics
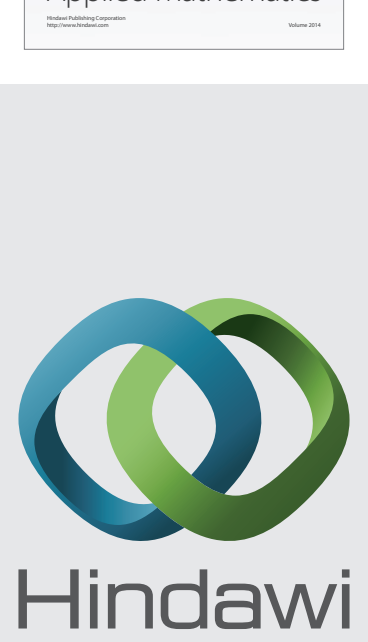

Submit your manuscripts at http://www.hindawi.com
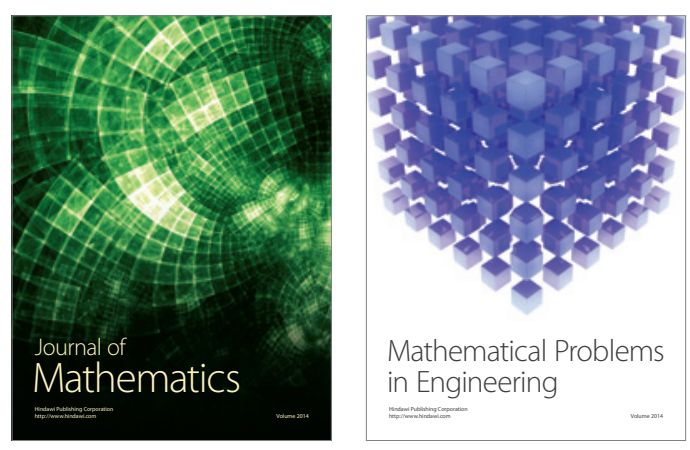

Mathematical Problems in Engineering
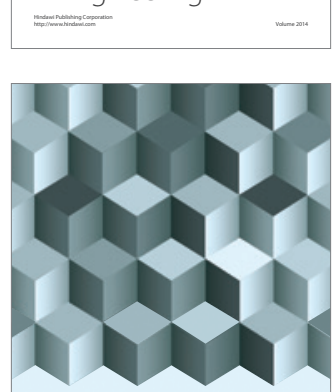

Journal of

Function Spaces
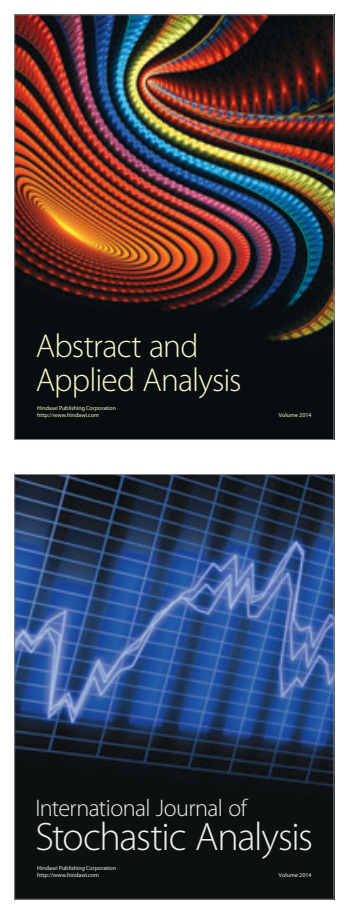

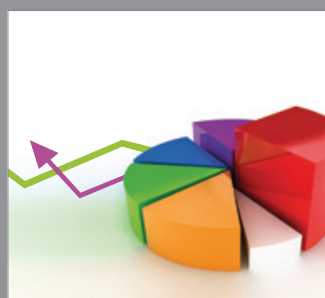

ournal of

Probability and Statistics

Promensencen
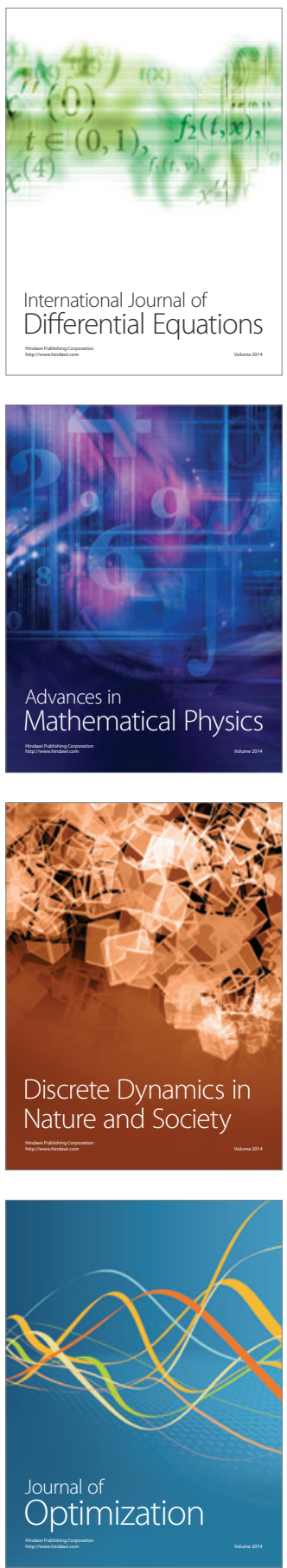